\section{Dependence of Electrical Conductance and Dielectric Gonstant upon Frequency in Mixtures of Strong Electrolytes}

THE properties of the ionic atmosphere in the new electrostatic theory of strong electrolytes are very im. portant in regard to reversible thermodynamic and irreversible processes. ${ }^{1}$ They enable us, for example, to develop a theory of the irreversible conductivity processes and of the irreversible mechanism that is involved in the viscosity phenomena in the case of a simple electrolyte. We have recently been able to find the general relation between frequency and electrical conductance and dielectric constant in mixtures. ${ }^{2}$ From our general equation of the force of relaxation one can now derive a detailed discussion of the quantitative limiting laws of the conductivity and the dielectric constant in mixtures of strong electrolytes. The special discussion-for example, of the problem of 3-ions and 4-ions and so on-is very complicated and will occupy some time. ${ }^{3}$

We thought it desirable, however, to give at once a theoretical explanation of the new experimental work done by Spaght." Spaght has inquired into the dispersion of the conductance of two mixtures of strong electrolytes. His results can be explained theoretically - in a qualitative manner at least-by a further generalisation of the calculations made by Bennewitz and Wagner-Küchler, who have worked out only the stationary case, where a relatively small quantity of ions of sort (3) are mixed with an electrolyte consisting of ions of sorts (1) and (2). Our calculations deal with the non-stationary case and give the expression for the mobilities and the dielectric constant of an electric field alternating with high frequency. Even in this simple case, the expressions for the electrical conductance and the dielectric constant are relatively complicated. We will not give this expression now, as it will be published shortly. 5

It would be very interesting to compare the theoretical results with further systematic experimental studies which will have to be carried out in the future. In the case of a simple electrolyte, this has been done successfully by many workers who obtain quantitative agreement with the theory of Debye-Falkenhagen. (See the monograph by Falkenhagen, loc. cit.) It should be possible to investigate not only the dependence on frequency of the electrical conductivity but also that of the dielectric constant effect by means of the method worked out by $M$. Wien. ${ }^{6}$ The latter effect is based on the fact that we have a phase difference between the field strength and the ionic velocity produced. That means an electric field produces two components of the electrical current; one of them has the same phase as the field strength and the other one is in phase with the differential quotient of the field with respect to the time or the Maxwell displacement current. The quantity of the displacement current is proportional to the dielectric constant. Hence we get an alteration of the dielectric constant (relatively to the dielectric constant of the pure solvent) which is dependent on the frequency of the field.

It is easy to see that we have to deal with an increase of the dielectric constant. Consider a quick displacement of a central ion; the consequence of the finite time of relaxation of the ionic atmosphere is that a quasi-elastic force will arise which will repel this central ion to its original position. This quasi-elastic binding between the ions means an increase of the dielectric constant. In the case of more concentrated solutions and relatively very high frequencies, there will be, of course, other interesting effects on the conductance and the dielectric constant, for example, the quantum mechanical forces, dipole effect, effect of solvation and so on.

One important point of view in the development of the theory of electrolytic solutions has yet to be considered. The Debye theory is applicable only in the case of sufficiently dilute solutions and gives the quantitative limiting laws. The electrostatic theory makes use only of the Coulomb forces between the ions. Not only are the electrostatic forces, which means the Coulomb forces and the forces of polarisation, significant, but also the quantum mechanical forces and the interionic dispersion forces are of great importance. The latter correspond to the van der Waals' forces in the case of gases and are not only of electrostatic nature but also of quantum mechanical nature in the sense of London, Eisenschitz and Margenau.

Taking into account these ideas one has to replace the interionic forces in vacuo by the corresponding forces in a solvent; that means that in the first approximation one has to substitute $e^{2} / D r$ for $e^{2} / r$ in the formulæ developed by the quantum mechanics, where $D$ is the dielectric constant and $e$ the electric charge.

It would be very interesting to extend the theory in this direction. We believe that it would be possible to develop a statistical treatment of more concen. trated solutions in the sense mentioned above. This is one of the most interesting problems in the field of electrolytic solutions.

Hans Falkenhagen. Walter Fischer.

Physical Institute, University, Cologne.

${ }^{1}$ A complete treatment of the modern theory of electrolytes and critical handling of the experimental data has been tried in the monograph "Elektrolyte" by H. Falkenhagen, Lelpzig, S. Hirzel, 1932. The English translation will be published in the series of monograp
edited by Prof. R. H. Fowler (Cambridge University Press).

H. Falkenhagen and W. Fischer, $P$ hys. $Z$., in press.

One of us (Fischer) will give the special discussion in a forthcoming dissertation.

5 A paper to be published in the Physikalisches Zeitschrift (1933). - A. Wien, Phys. Z., 31, 793; 1930. 32, 183; 1931. Ann. Phys., (s) 11,$429 ; 1931$.

\section{Infra-Red Absorption of Quartz}

Is 1895 Merritt, ${ }^{1}$ observing the infra-red absorption in the $2 \cdot 9 \mu$ region of a beam passing at right angles to the axis in crystalline quartz, found a definite difference when the radiation was polarised in planes at right angles and parallel to the axis. That is, the ordinary and extraordinary rays respectively were differently absorbed. The absorption of a beam parallel to the axis was, as was to be expected, similar to that of the ordinary ray in the first case. Koenigsberger ${ }^{2}$ in 1897 made like observations.

$I$ am unaware of any subsequent investigation of this effect in this region and wish to direct attention to its importance, especially as the absorption bands for radiation passing parallel to the axis may be required to serve as standards of wave-length in the infra-red. Dreisch ${ }^{3}$ has published a curve showing four bands in this region though he only states the wave-lengths of three of them.

I have recently examined specimens of quartz with

No. 3294, VoL. 130] 